\title{
Geostatistical Analysis for the Study of Relationships between the Emotional Responses of Urban Walkers to Urban Spaces
}

\author{
Ihab Hamzi Hijazi, An-Najah National University, Nablus, Palestine \\ Reinhard Koenig, ETH Zurich, Zurich, Switzerland \\ Sven Schneider, Bauhaus-Universität Weimar, Weimar, Germany \\ Xin Li, School of Urban Design, Wuhan University, Wuhan, China \\ Martin Bielik, Bauhaus-Universität Weimar, Weimar, Germany \\ Gerhard Norbert Johannes Schmit, ETH Zurich, Zurich, Switzerland \\ Dirk Donath, Bauhaus-Universität Weimar, Weimar, Germany
}

\begin{abstract}
The described study aims to find correlations between urban spatial configurations and human emotions. To this end, the authors measured people's emotions while they walk along a path in an urban area using an instrument that measures skin conductance and skin temperature. The corresponding locations of the test persons were measured recorded by using a GPS-tracker $(n=13)$. The results are interpreted and categorized as measures for positive and negative emotional arousal. To evaluate the technical and methodological process. The test results offer initial evidence that certain spaces or spatial sequences do cause positive or negative emotional arousal while others are relatively neutral. To achieve the goal of the study, the outcome was used as a basis for the study of testing correlations between people's emotional responses and urban spatial configurations represented by Isovist properties of the urban form. By using their model the authors can explain negative emotional arousal for certain places, but they couldn't find a model to predict emotional responses for individual spatial configurations.
\end{abstract}

\section{KEYWORDS}

Emotion, Geo-Statistical Analysis, GIS, GPS, Isovist, Point Pattern Analysis, Regression Analysis, Space Syntax Theory, Urban Design 


\section{INTRODUCTION}

A procedure for the evaluation of urban design proposals from a human centered perspective is important in the planning of cities. One of the key steps in the planning of cities is the definition of urban form in which decisions about shape, dimensions and arrangements of buildings are made. These decisions have a relatively long lasting impact on the future development of a city, because once built, streets and buildings are hard to change. It is, therefore, important for planners to assess the implications of their decisions as early and as comprehensively as possible. A better understanding of the impact of urban form on the behavior of people can improve the design of cities (Hillier, 1996; Gehl, 2010).

A relatively new and promising design method is termed evidence-based design, which aims to evaluate a certain level of design quality by measurable criteria (Chong et al., 2010, p.1 ff.). In our context, an important aspect is to predict the most likely behavioral responses to a specific design proposal. Capturing the most likely behavioral reactions is crucial, because cities are typically designed for long time spans and must satisfy the needs of many individuals. Given the fact that individual actions are probably never fully rational and predictable and depend on many factors that are not a direct consequence of urban design (such as prior personal experience, cultural background, health, wealth), it is only possible and useful to identify the potential for promoting or avoiding certain behavioral reactions. In the framework of this paper we investigate emotional responses to space and assume that our emotions are an important aspect that influence behavior (Zajonc, 1984, Winkielman, Berridge, \& Sher, 2011).

We start from the hypothesis that people have different emotional responses to different urban spaces. Based on this we structured this study in two phases: In a first step we measure human emotional responses in space using a sensor-wristband (Smartband), developed by Bodymonitor (Papastefanou, 2013a) in combination with a GPS-tracker. In a second step we look for correlations between the measured emotional responses and Isovist properties (Batty, 2001; Benedikt, 1979; Turner \& Penn, 1999).

As a methodological basis for both phases we present a GIS-based spatial analysis method for evaluating the collected data. In the analysis we examine the probability that the distribution of emotion values occurs by chance, where the areas of clustering of high and low values of emotional response are, and finally if this clustered arousal in certain locations is caused by Isovist properties.

An Isovist describes the part of an environment that can be seen from a single observation point (Benedikt, 1979). Various parameters are derivable from an Isovist, such as the area, the perimeter, compactness and occlusivity. The area of an Isovistis a measure of how much one can see from a certain vantage point. The property of compactness describes the relationship between area and perimeter in relation to a perfect circle. It indicates how complex or compact the field of view is. Occlusivity indicates the amount of open edges. An open edge denotes an edge line of the visual field which is not defined by physical boundaries (e.g. walls). Occlusivity is small in locations in which only a few or no views into other parts of the spatial configuration are possible (e.g. when occlusivity $=0$, the space is entirely enclosed). We use Isovist properties for this study, because they can be computed by using building footprints only, which are available for every location via Open Street Maps.

In a wider context, the study intends to contribute towards a Computationally Aided Evidence Informed Design process (CEID). The main idea of CEID is that features of the urban form are derived directly from the specific design proposal, and therefore no a priori categorizations are necessary. Here we would like to add the ability to estimate human emotional responses to urban spatial configurations, which we estimate using Isovist properties. There are studies that cover other ways of analyzing urban form according to aspects such as visibility (Bittermann and Ciftcioglu 2008), accessibility (Hillier, 1996; Sevtsuk, 2010) or daylight (Ratti et al, 2003; Compagon 2004). A key question in this context is how to determine if there are significant correlations between the measures derived from the computational analysis, and identifiable human behavioral responses to urban form. 
If such correlations can be shown, these measures could then be utilized to evaluate and improve design proposals. The components necessary for establishing a CEID process are shown in Figure 1. The development of a CEID, however, will require more studies of man-environment relationships.

\section{STATE OF THE ART}

Several studies exist in which computed measures of urban space have been successfully correlated to certain human behavior. For example, Hillier \& Iida (Hillier and Iida, 2005) have shown that centrality measures of the street network (which is based on the geometry of the open space generated by the shape and arrangement of buildings) have a significant impact on movement patterns. These movement patterns in turn have a significant impact on the use of public space, given that commercial uses are generally located at more frequented locations (Hillier, 1996; Sevtsuk, 2010).

Furthermore, several studies suggest that properties of the visual field play an important role in how complexity (Franz \& Wiener, 2008; Meilinger, Franz, \& Bülthoff, 2012), spaciousness (Stamps, 2009), and orientation (Conroy et al, 2010; Wiener et al, 2011) are perceived, to name just a few.

Based on progress in neuroscience in the past decade, a separate research stream has emerged that seeks insights for the field of architectural design using methods used in neuroscience (Academy of Neuroscience for Architecture, 2013). Important study areas in this field include the effects of the form and function of architectural environments on human health (Edelstein and Macagno, 2012) or on way finding strategies (Edelstein et al., 2008). Both studies (Edelstein and Macagno 2012; Edelstein et al., 2008) used virtual environments which made it possible to carefully control the studied parameters. But because it is not very clear how these correlate with real space, for example with regard to the incorrect estimation of distances and angles (Peters and Richter, 2008), we have chosen to focus on undertaking studies in real world environments.

Figure 1. The basic framework for Computationally Aided Evidence Informed Design

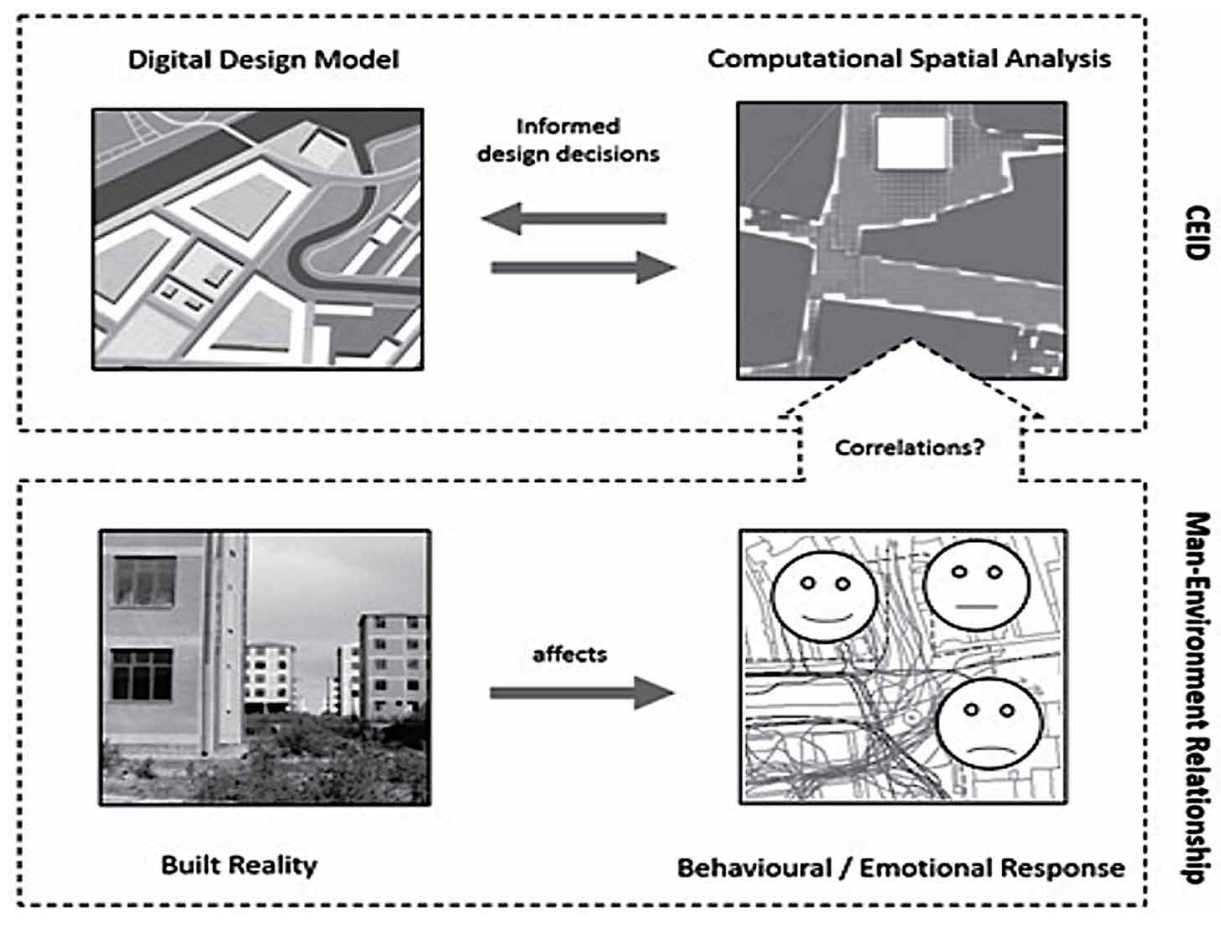


The model we use in this study for measuring emotions is based on the concept of cognitive appraisal that categorizes a relatively complex set of secondary emotions (Russell, 1980). Secondary emotions are those that have a major cognitive component. They are determined by both their level of arousal (low to high) and their valence (pleasant to unpleasant). Figure 2 shows a model of how various secondary emotions can be located inside a coordinate system.

Hogertz (Hogertz, 2010) studied the emotions of urban walkers $(n=31)$ in Lisbon collecting them using Smartbands and GPS-tracking. Hogertz analyzed the emotional responses, compared to the retrospective emotional states of the subjects, by visual inspection. He concluded that "specific emotional significance can be measured reliably by recording a person's EDA [electrodermal activity] variations while walking". From his examinations he found that there is primarily a relationship between negative emotional responses and certain locations.

In another study aimed at improving barrier-free-planning for handicapped people, the emotions of blind people and those with walking disabilities (in total $n=39$ ) were measured using Smartband technology and GPS-tracking (Bergner et al, 2011). The study revealed that different sectors in a part of the pedestrian precinct of Kaiserslautern, Germany, were associated with various stress-levels for disabled people.

A further analysis was undertaken in the main promenades of Alexandria, where individual stress reactions $(n=7)$ were identified using a promising workflow (Bergner et al., 2013). They coupled datasets from a GPS-Tracker, camera, and Smartband, and identified stress phases over the route taken and extracted movie snippets of the relevant sections. To visualize the results, all the individual stress points were aggregated in a heat map, using point density analysis that showed stress hotspots.

In another study, neural imaging using EEG was used to map human responses to spaces (Mavros, Coyne, Roe, \& Aspinall, 2012; Roe, Aspinall, Mavros, \& Coyne, 2013). The authors describe an experiment where the affective (emotional) states of test persons were measured while moving

Figure 2. A model for secondary emotions determined by level of arousal (low to high, respectively mild to intense) and their valence (pleasant to unpleasant). Figure adapted from Russell (1980).

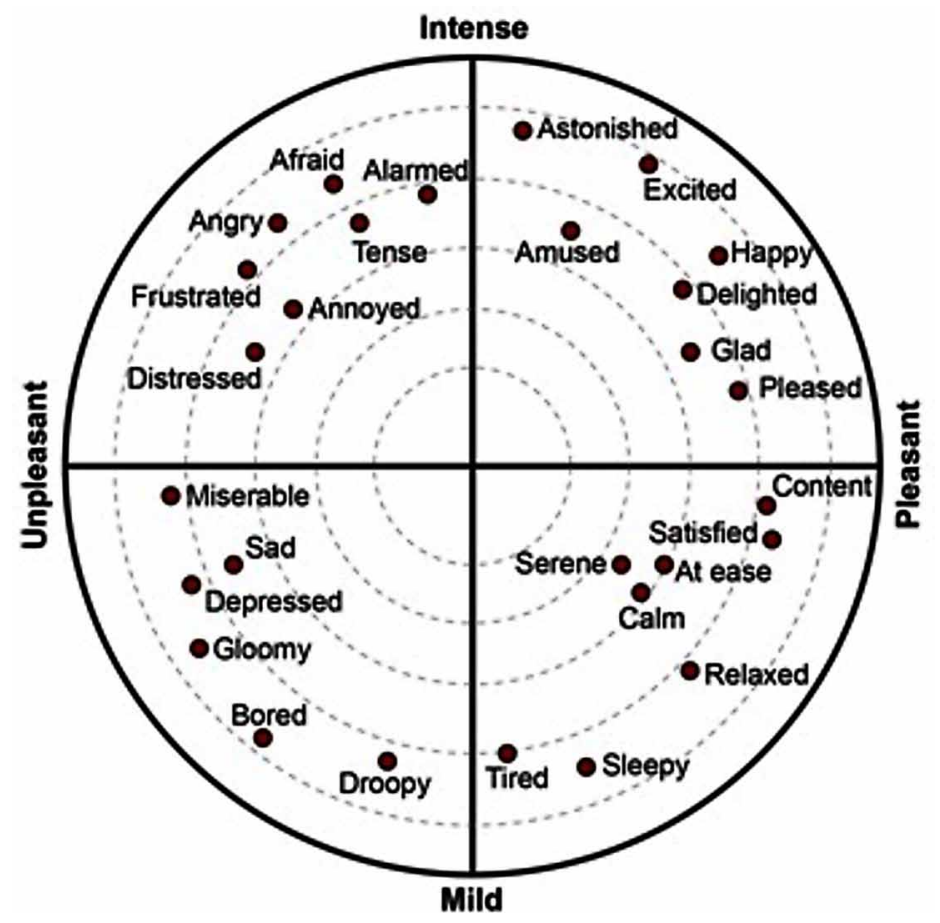


through open spaces in Edinburgh. They used a light weight, high performance laptop, wireless EEG and a GPS unit. The collected data were analyzed by mapping them to the defined path in terms of excitement and frustration levels.

All the above studies that measure emotional response show that there are locations in the urban realm that elicit significant emotional responses. But none of them tested whether individual perceptions of spaces correlated with those of other individuals to ascertain whether certain spatial configurations have a general effect on human emotions. Also, none of the studies described above tested whether there is a relationship between people's emotion and Isovist field parameters. These are the areas in which this study aims to make a contribution.

\section{Data Collection}

The Smartband (Papastefanou, 2013a) offers a mobile means of measuring skin conductivity (SC) as well as skin temperature. The electrodermal activity and temperature is measured by metallic electrodes, which are located near the wrist on the inner side of the forearm. The Smartband records one dataset per second and stores it on an internal storage medium. The wristband is lightweight and relatively comfortable to wear, and doesn't affect the mobility of the test person.

Using the GPS-tracker we can assign locations to the emotion-data we collect. We used a Qstarz BT-Q1000eX tracker, which represents a good compromise between cost and measurement precision (Stahli and Keller, 2013). It is approximately the same size as a matchbox and can easily be placed in a pocket or carried by hand for better signal reception. We used it in $1 \mathrm{~Hz}$ mode to record position data every 5 seconds ( $10 \mathrm{~Hz}$ would be possible, too). As the Smartband data have a higher temporal resolution, the results were aggregated afterwards to match the 5-second recording interval of the GPS device.

Before the test persons were sent on their respective ways, the Smartband measurement was calibrated using a standardized procedure (Papastefanou, 2013a) for each person with the aim of ensuring that the data collected for each person's skin conductivity responses were comparable. The measured data of the urban routes are stored separately in the Smartband and in the GPS-tracker and the two data sets linked afterwards via their timestamps.

The empirical study took place from 14 - 22 October 2013 and the route of the urban walk was predefined: all test persons followed the same path shown in Figure 3. The length of the path was approximately $2.2 \mathrm{~km}$, for which the test persons needed approximately $30 \mathrm{~min}$ when walking. We chose a route in the district of Oerlikon in the city of Zurich that encompasses different urban areas, e.g. a newly built office area, a quiet residential area and a busier local center in the Oerlikon district as shown in the photographs in Figure 3.

Fourteen participants (students and lecturers) took part in the study, and one dataset had to be excluded as invalid because the Smartband had been used incorrectly. Consequently, our final test results include $n=13$ valid datasets, of which 12 were male and 1 was female.

We gave each test person the following description and instructions, including the map in Figure 3: Choose a time for your walk on our list (morning or afternoon); Pick up the GPS-tracker and the 'SmartBand' at the department for your time slot; Go to 'Max-Bill-Platz'; Fill in the start time of the walk on this document; Turn on the GPS-tracker $(1 \mathrm{~Hz}$ position) and press the red button; turn on the 'SmartBand'; Take your first photo in the direction in which you are walking; Follow the route on the map; At the positions marked on the map (arrows), take a photo (in your walking direction) and press the red button on the GPS-tracker; At Oerlikon Markt platz take three photos as indicated on the map; When you arrive at Oerlikon station, take a final photo; press the red button on the GPS-tracker; turn off the GPS-tracker and the 'SmartBand'; Bring back the GPS-tracker and the 'SmartBand'.

Pressing the red button on the GPS-tracker marks that specific location. The participants were asked to take photos at the marked locations, because we wanted them to remain at a location for a few seconds to test whether we could get better or different measurements for emotional responses. 
Figure 3. The participants were given a map showing the path (red dotted line) they were asked to follow (base map from OpenStreetMap). At places marked with an arrow they we are asked to take a photo and wait at least 5 seconds to enable the Smartband to take adequate measurements. The photographs on the right side show some characteristic street views.

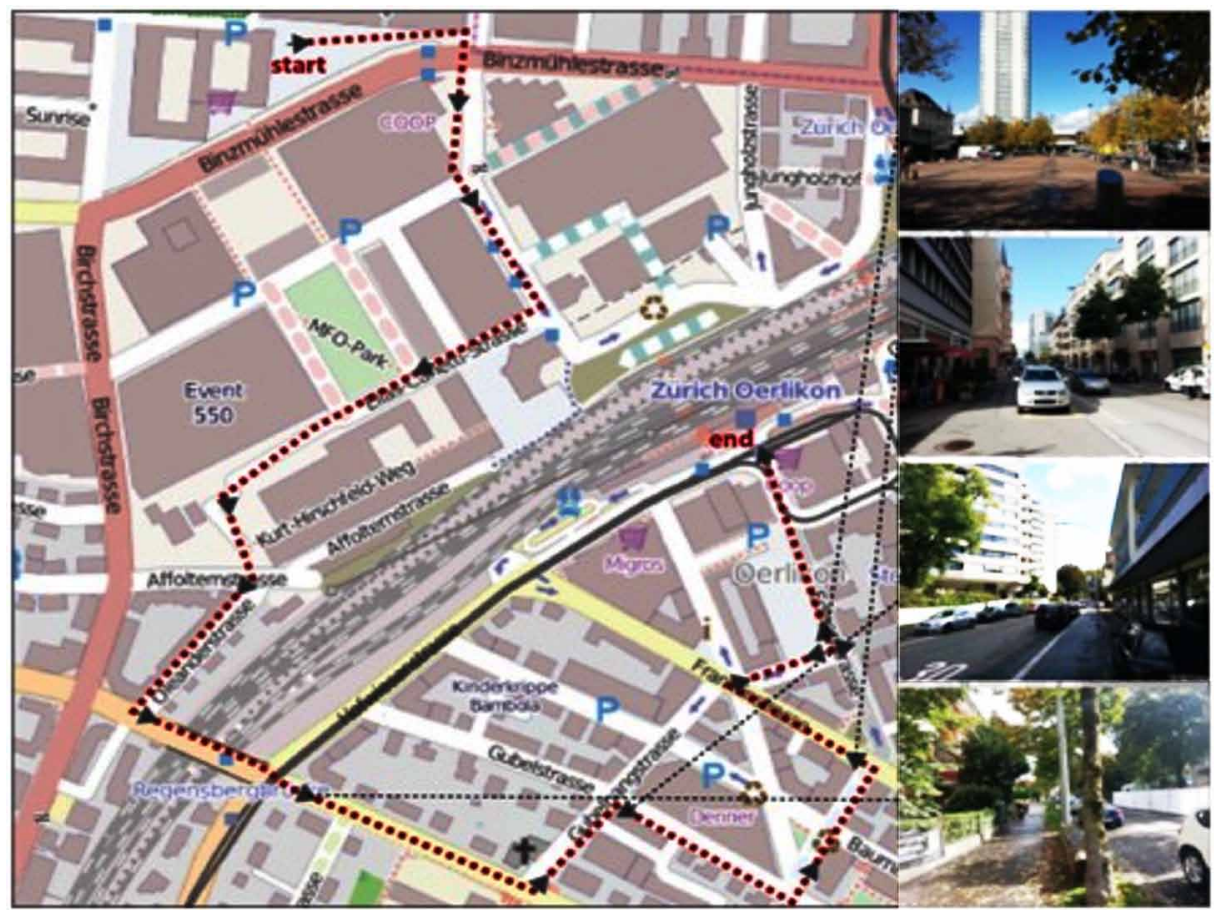

The subsequent analysis of the data showed that this had no significant effect, and we will omit this in future studies.

From the data collected we can derive emotional responses as described by the secondary emotions model (Russell, 1980) shown in Figure 2. The company Bodymonitor processed the raw data collected by the Smartband and analyzed them with respect to four activations: Negative arousal, positive arousal, balanced, and retraction as described in Table 1. Evidence for the validity and reliability of this method of categorizing emotions in four types (Table 1) can be found on the

Table 1. Typology of emotional impulses. Table reproduced from BodyMonitor (Papastefanou, 2015).

\begin{tabular}{|l|l|l|l|}
\hline \multicolumn{1}{|c|}{$\begin{array}{c}\text { Emotional Activation } \\
\text { (Impulse) }\end{array}$} & \multicolumn{1}{|c|}{ Emotional System } & \multicolumn{1}{|c|}{ Stimuli } & Corresponding "Feelings" \\
\hline Positive & $\begin{array}{l}\text { Orientation, positive } \\
\text { expectation }\end{array}$ & Appetitive stimuli & Interest, surprise, joy \\
\hline Negative & Flight, fight & Aversive stimuli & $\begin{array}{l}\text { Fear, anger, disgust, stress, } \\
\text { rage }\end{array}$ \\
\hline Balanced & Rewarding & Consumptive stimulus & $\begin{array}{l}\text { Satisfaction, attention, } \\
\text { vigilance, well-being, } \\
\text { "flow" }\end{array}$ \\
\hline Retraction & "Shut down" & No activating stimulus & $\begin{array}{l}\text { Tired, mentally withdrawn, } \\
\text { bored, sadness, defense }\end{array}$ \\
\hline
\end{tabular}


Bodymonitor website (Papastefanou, 2013a). The precise details of the method are, however, not explicitly published.

\section{ANALYSIS \\ Data Preparation}

The GPS and emotion sensor data were received as csv files. The data were imported into ArcGIS to join the two separate data sets and overlay the data points on the map of Zurich Oerlikon (Figure 4). For each test person, the GPS points and emotional data were linked via their synchronized time stamps. The joined data were aggregated in one feature class, representing events locations (Figure 4).

The data of the locations with associated emotional responses were re-projected to the CH1903 LV03 (Swiss Coordinate Reference System) to make sure that the distortion in distance is minimal. Figure 5 shows an excerpt of a table with the joined data features.

\section{Basic Statistical Analysis}

Based on our points along the path we were able to derive some indications of patterns: Getis-Ord General G, and Getis-Ord hot-spot analysis ( $\mathrm{Gi}^{*}$ ) functions were used in the following analysis (Getis, 1991; Mitchell, 2005). These were then calculated and visualized using ArcGIS. To begin with, we analyzed the probability that the distribution of the emotion values occurs randomly.

The aim of the first part of the analysis is to establish two things: firstly, whether the emotion values are clustered; and secondly, the distance over which the clustering occurs. The values of distances between the data points are critical for identifying how compact the groupings are. This is used later to perform hot-spot analysis. This analysis was conducted using General G statistics to show whether the data distribution is random or not. The General G statistics method measures the

Figure 4. The points show the locations of recorded emotional responses along the urban route

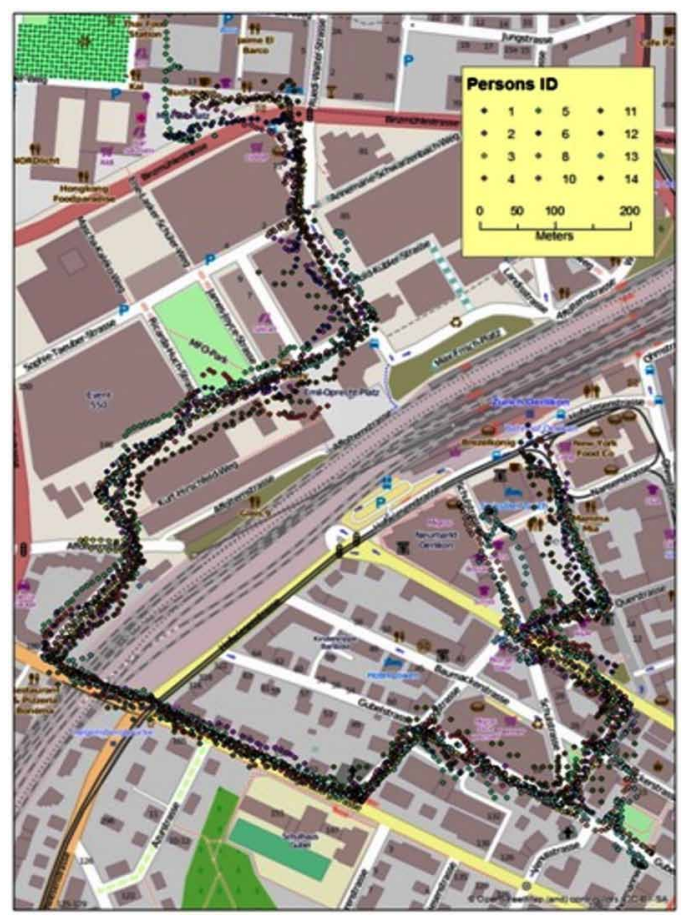


Figure 5. Screenshot of emotion data features with its associated table

\begin{tabular}{|c|c|c|c|c|c|c|c|c|}
\hline \multicolumn{9}{|c|}{ EmotionCollectionSwissProj } \\
\hline & OBNECTID* & SHAPE * & LOCAL_DATE & LOCAL_TIME & bmcase & index_1 & neg_arousa & pos_arousa \\
\hline - & 1 & Point Z & $10 / 15 / 2013$ & $13: 4: 46$ & 1 & 1 & 0 & 0 \\
\hline & 2 & Point Z & $10 / 15 / 2013$ & $13: 4: 52$ & 1 & 2 & 8.177783 & 0 \\
\hline & 3 & Point $Z$ & $10 / 15 / 2013$ & $13: 4: \$ 7$ & 1 & 3 & 0 & 12.217996 \\
\hline & 4 & Point Z & $10 / 15 / 2013$ & $13: 5: 2$ & 1 & 4 & 5.280245 & 0 \\
\hline & 5 & Point Z & $10 / 15 \sqrt{2013}$ & $13: 5: 7$ & 1 & S & 0 & 0 \\
\hline & 6 & Point Z & $10 / 15 / 2013$ & $13: 5: 12$ & 1 & 6 & 0 & 7.962041 \\
\hline & 7 & Point Z & $10 / 15 / 2013$ & $13: 5: 17$ & 1 & 7 & 7.848558 & 0 \\
\hline & 8 & Point Z & $10 / 15 / 2013$ & $13: 5: 22$ & 1 & 8 & 2.003027 & 0 \\
\hline & 0 & Daind 7 & Anteronse & $12 \cdot 5 \cdot 37$ & 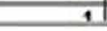 & 01 & 1 eenas? & n \\
\hline
\end{tabular}

concentration of high or low values in a defined area and compares this to how high or low the rest of the values are. Here we apply it to the entire study area rather than a localized area.

Since one feature's values has no effect on the value of the nearby features, the analysis was undertaken using fixed distance band settings and the function was run several times to determine how location affects clustering. As each test person was recorded individually, and at different times, one person's emotional response had no effect on that of another person, i.e. the emotional response of person $\mathrm{A}$ in one space has no relationship to the emotional response of person B in the same space.

Using ArcGIS G tool, a range of distances were tested and the highest z-score was found using $23.5 \mathrm{~m}$. The null hypothesis for this analysis was: the emotion-values for our test persons are randomly distributed across the study area. Given the z-score of 2.87, and the fact that the observed G-index is higher than the expected G-index, there is a less than 5\% likelihood that this highly-clustered pattern has occurred by chance. This allows us to safely reject the null hypothesis, in turn confirming that the positive arousal values are clustered.

The G-index analysis was undertaken for negative arousal using a range of distances and the result shows significant clustering of the negative arousal value at a distance of 11 meters. Given the z-score of 1.96 at 11 meters distance, there is a less than 5\% likelihood that this highly-clustered pattern could be the result of random chance. This too confirms that the null hypothesis can be rejected. Negative arousal is clustered.

\section{Mapping}

The aim of this mapping is to show where high and low values are clustered. The Gi* statistics uses both the locations and the values for pattern calculation. It compares the values of each feature (the positive or negative arousal of a test person at a specific location) with the neighboring feature values within a user-specified distance. The values for each feature are then color-coded to show high and low value clusters of positive or negative emotional arousal(Figure 6 and Figure 7). The results show where emotional response clusters arise in space for comparison with spatial configurations, which may be responsible for these clusters.

The collected data contains separate fields for positive and negative emotional arousal values. The $\mathrm{Gi}^{*}$ statistics were conducted on the data with a fixed distance band of 23 meters for the positive values and 11 meters for the negative values. The distance values were determined from the General $\mathrm{G}$ statistics (Table 2 and Table 3). Figure 6 shows the cluster of high and low values for positive arousal and Figure 7 for negative arousal. The dark blue areas represent clustering of low values and the red areas represent clustering of high values. This result provides a clear indication of where similar high and low values of both positive and negative emotional arousal are clustered with a significant level of confidence above 95\% (Figure 6 and Figure 7).

To validate the analysis results, we undertook a manual check to make sure that the clustering of similar emotional arousal values is actually the product of different test persons. For three cluster 
Figure 6. Locations of positive emotional arousal are color-coded by Z-scores. Locations surrounded by locations with similar high or low values are shown in red or blue. The locations P1 - P6 were used for the photographic comparison below. The areas $\mathrm{C} 1-\mathrm{C} 3$ are used to investigate the number of different test persons inside a cluster.



locations C1-C3 (Figure 6), we assigned the ID of the test persons to the data points. The results show that the data points in the tested clusters belong to more than 10 out of the 13 test persons (Figure 8).

\section{Interpretation of the Basic Statistical Analysis}

Since the clusters of positive and negative arousal shown in Figure 7 and Figure 8 are based on data from different test persons walking along the path at different times and days, we can assume that influencing factors such as noise, weather or the individual's respective mood or personal background (e.g. whether they grew up in a city or in the countryside) etc. can be neglected for the interpretation of the results.

In an initial inspection of the different locations where we found clusters of positive and negative emotional arousal values, we looked at the pictures taken by the test persons during the walk.

In some areas, clusters of positive and negative emotional arousal are located close to one another (Figure 6 and Figure 7). For example, P2 (second photo in Figure 9) and N1 (first photo in Figure 10) are close to each other. A possible explanation is that emotional response is not caused primarily by a certain spatial situation but rather by a changing sequence of spaces. If there are no changes in the environment our emotions are more or less balanced and simply take in impressions.

This interpretation is supported by the fact that positive or negative emotional arousals are often stimulated as one enters a new spatial situation, for example after turning a corner and looking into the next street or square (Figure 6 and Figure 7). This observation is supported by measurements of emotional responses where the locations of arousal spots along a path depend on which way the test person is walking along the pre-defined path (Hogertz, 2010). 
Figure 7. Locations of negative emotional arousal are color-coded by Z-scores. Locations surrounded by locations with similarly high or low values are shown in red or blue. The locations N1 - N3 were used for the photographic comparison below.

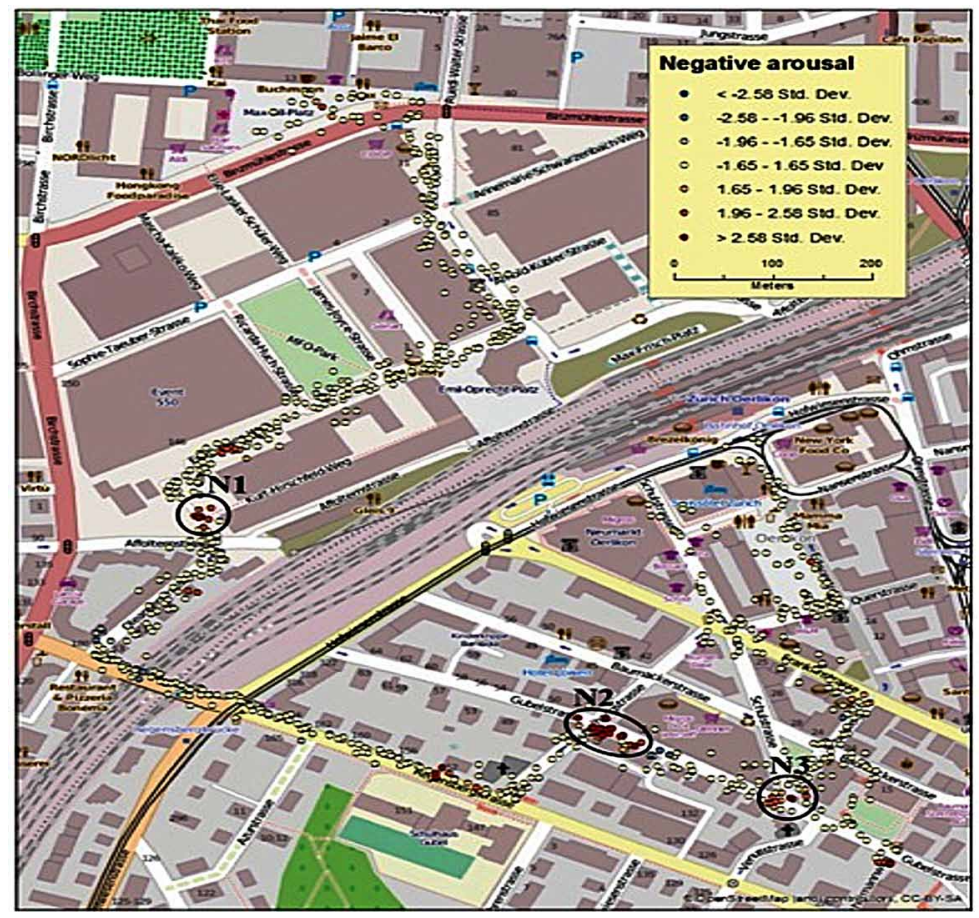

Figure 8. Locations of positive emotional arousal for the clusters C1 - C3 showing the points labeled with the test person's IDs

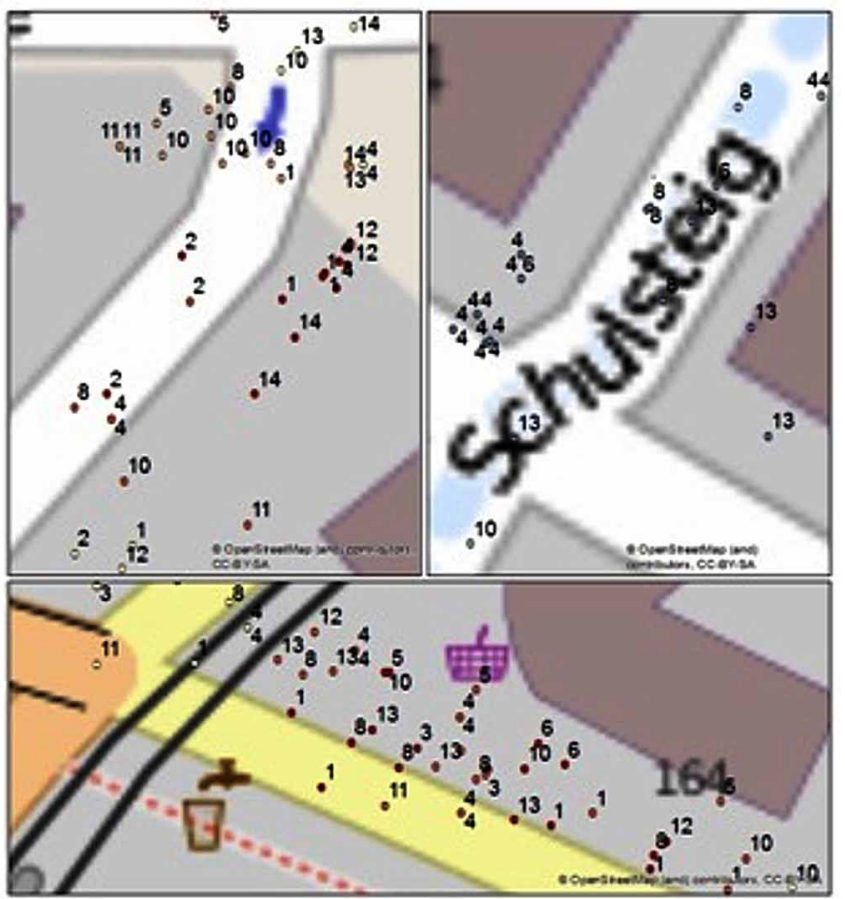


Figure 9. Photos of spatial situations at locations of clusters with positive emotional arousal values. From top left to bottom right the pictures are from clusters $\mathrm{P} 1-\mathrm{P} 6$.
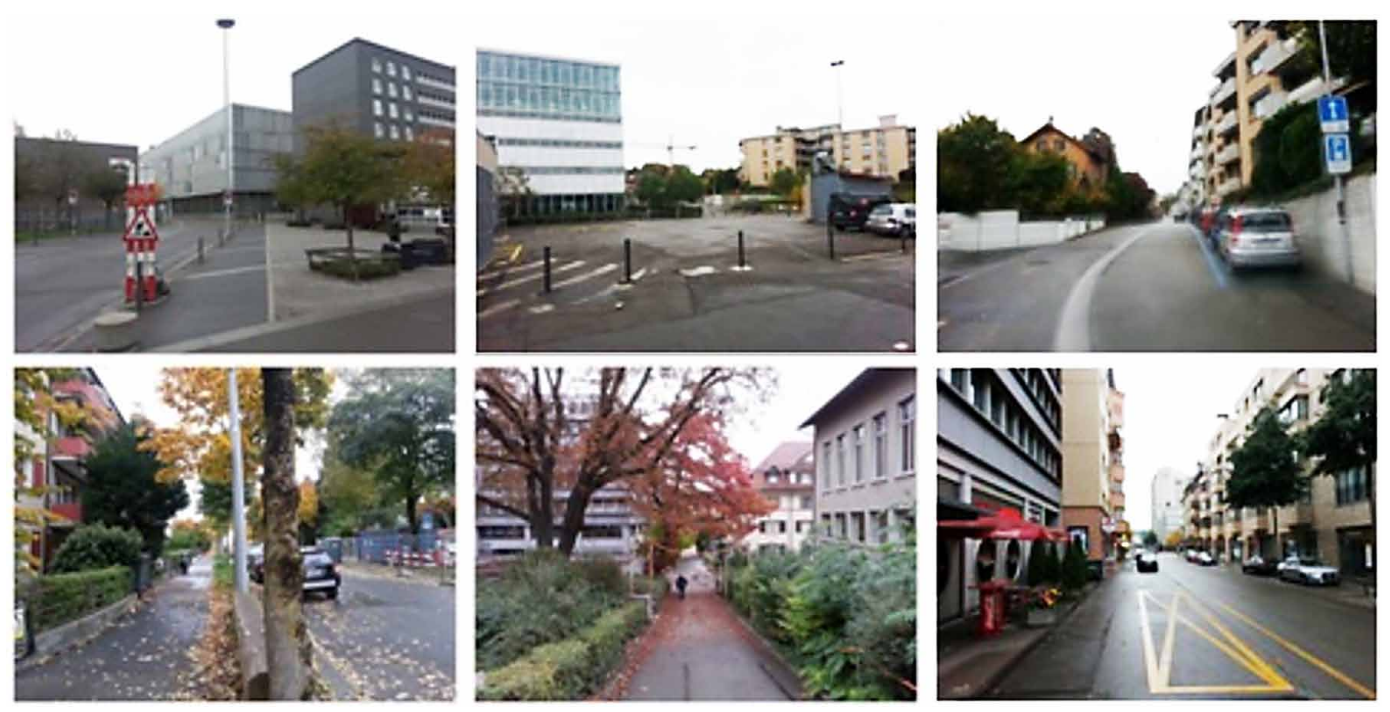

Figure 10. Photos of spatial situations at locations of clusters with negative emotional arousal values. From top left to bottom right the pictures are from clusters $\mathrm{N} 1-\mathrm{N} 3$.
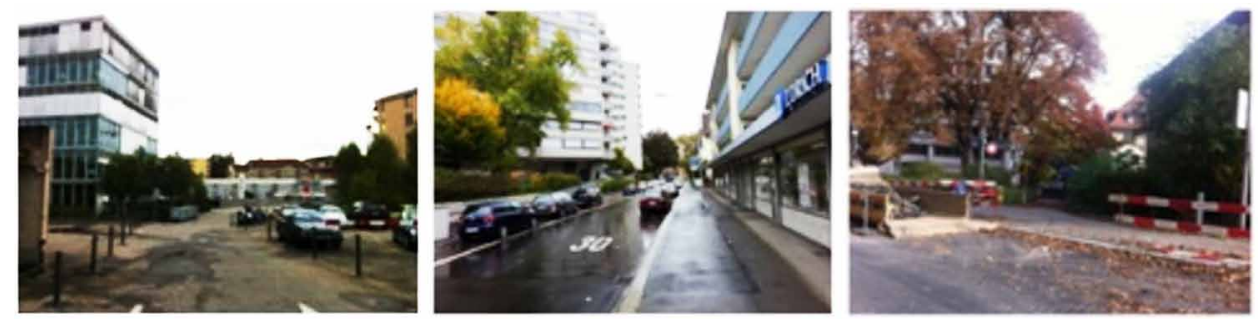

When we compare the photos of the clusters of positive (Figure 9) and negative (Figure 10) emotional arousal, we see situations that contain various constellations of trees in both. Also the style of the houses or the façades offers no clear indicator for a positive or negative response.

\section{Predictive Model}

In the second part of the paper, we develop a model for predicting emotional response of pedestrians in urban space. To this end, we look for correlations between computed Isovist properties and the emotional responses. As described in the introduction, this is useful in order to make statements about the emotional impact of urban design, such as the arrangement of buildings or the sizing of public squares, that can inform the design process. For our model we use the properties of $2 \mathrm{D}$ Isovists to predict emotional responses. This means that we consider the Isovist properties as independent variables, and the people's positive and negative arousal as dependent variables. The following Isovist properties were used: Area, perimeter, compactness and occlusivity.

Prior to conducting the regression analysis, Isovist properties were calculated for each point along the defined route that denotes the test person's location and their respective positive and negative arousal values. Figure 11 shows an exemplary sequence of 60 degree Isovists along the path. Points 
Figure 11. Top: Isovists along the path; Bottom: Sequence of Isovist properties (area, compactness, occlusivity) along the path
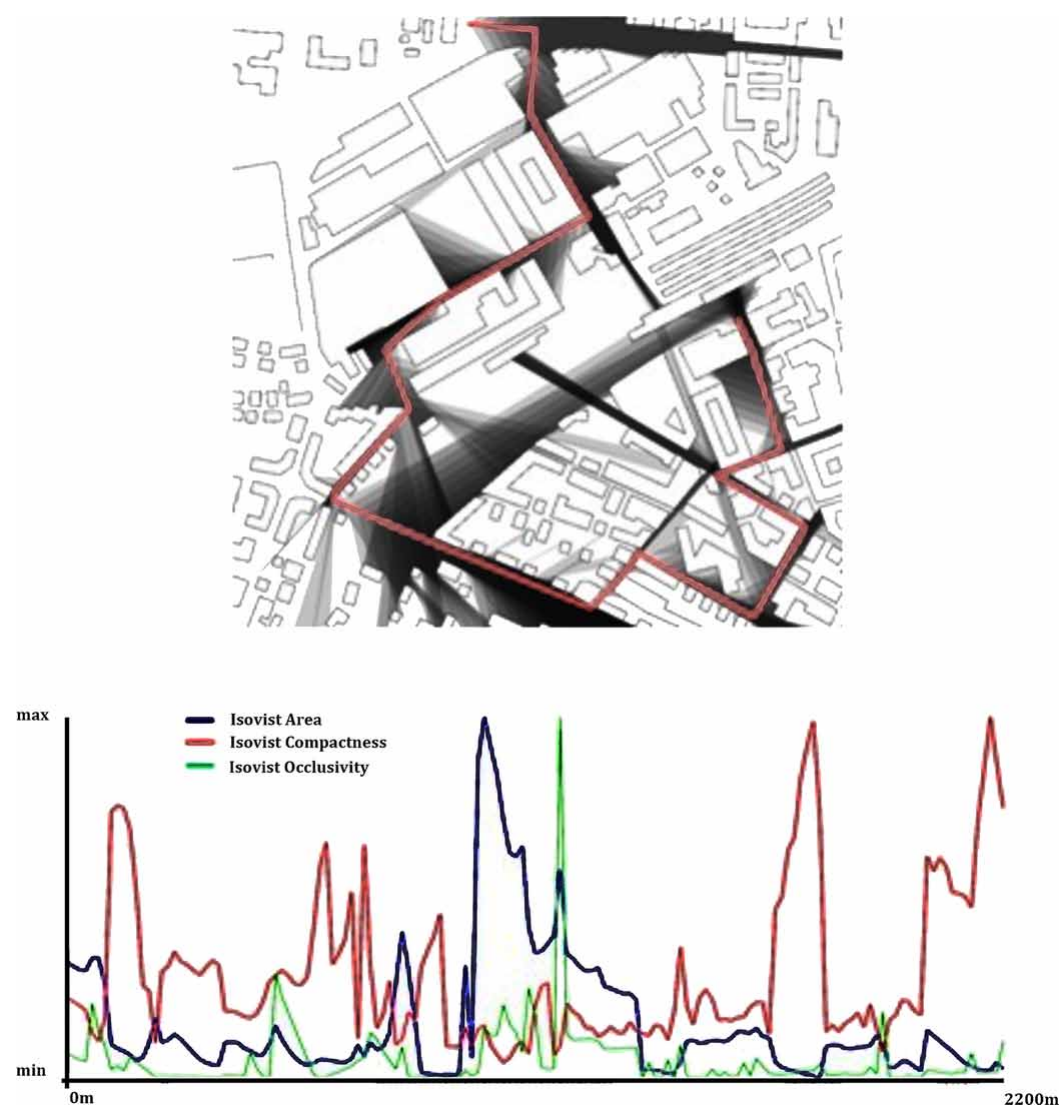

that were located inside buildings (Figure4) because of inaccurate GPS measurements were deleted. We calculated all Isovist properties for view fields of both 360 and 60 degrees.

From the analyses described so far, and especially the clusters of similar arousal values shown in Figure 6 and Figure 7, we expect that changes in a sequence of spaces along a path may be a reason for positive or negative emotional arousal. Consequently, it would be valuable not only to test static values for certain points of view but to investigate how the measured responses change along the path. The graph in Figure 11 shows such a sequence of changes of measurements for the Isovists.

To develop our predictive model, we start with the following hypothesis: There are certain spatial configurations which have a tendency to elicit specific emotional responses. The spatial configurations are quantified using Isovist properties.

\section{The Formal Model}

A logical breakdown for the model starts by calculating the Isovist properties that are considered as independent variables. A regression equation was developed to predict the people's positive and negative arousal values:

$\mathrm{Y}=\mathrm{b}_{0}+\mathrm{b}_{1} \mathrm{X}_{1}+\mathrm{b}_{2} \mathrm{X}_{2}+\mathrm{b}_{3} \mathrm{X}_{3}+\ldots+\mathrm{b}_{\mathrm{n}} \mathrm{X}_{\mathrm{n}}$

where $X_{i}$ is an Isovist parameter and $b_{i}$ is the weight that represents the influence of Isovist parameter $i$ to explain positive or negative emotional arousal $Y$. Using this regression model we can estimate the 
coefficients of the linear equation, involving one or more independent variables (Isovist properties) that best predict the value of positive and negative arousal.

\section{Identifying Key Variables}

For each case mentioned above, several trials were conducted to find out which of the 8 variables (Isovist properties estimated over 60 or 360 degrees) are driving the process. Accordingly, several trials were conducted as follows to obtain the most appropriate variables that best predict the value of positive and negative arousal.

Step 1: All variables (8 Isovist properties) were used in the model. This step provided an idea of the significance of each variable in the model, and helped in excluding variables with no significance.

Step 2: Each variable was used on its own in the model, to ascertain its proportional contribution to the dependent variable (people's positive or negative arousal value). We excluded independent variables that were not highly correlated with the dependent variable.

Step 3: Several trials were performed using only the most significant variables based on steps 1 and 2. This step included testing more than one variable sequentially in the model (using 1,2, then 3 and 4 variables) and testing the most appropriate model that best predicts emotional arousal using the following criteria.

The value of adjusted R-squared of the model should be increased by the inclusion of a variable. The greater the value, the better the model.

t-value of the coefficients, using $95 \%$ confidence level, should be greater than 2 . The greater the value, the better the results.

Based on the above principles, only two variables were found statistically significant for negative arousal and three for positive arousal. These variables were occlusivity with a60 degree field of view and perimeter with a 360 degree field of view for negative emotional arousal; and compactness with a 360 degree field of view, perimeter with a 60 degree field of view, and occlusivity with a 60 degree field of view for positive emotional arousal.

\section{GWR MODELING RESULTS}

Using the above Equation (1) a global regression model was created assuming that the relationship between variables is static over space. However, the Koenker test statistics (Burnham et al., 2002) indicate statistically significant non-stationary results.

Geographically Weighted Regression (GWR) (Fotheringham et al., 2003) is a method for exploring non-stationary spatial results. It is another type of regression with a local regression model, where Equation (1) is calculated for each feature using nearby features rather than all features in the dataset, thus allowing the relationships to change in response to spatial sequences. After we have found the properly specified model using the ordinary least square method implemented in the previous section, we use GWR, resulting in a better model (the $\mathrm{r} 2$ is increased and AIC (Akaike Information Criterion) is decreased). This means that allowing the relationship to vary with progression through space improves the model.

The GWR model is able to change with movement through space to reflect the influence of spatial location, resulting in a variety of summery statistics for many locations. This differs from a global model where one single summery statistic is computed for the whole area. GWR uses a series of inputs on the variables to change the relationship between the variables so that they are directly compared over area (GWR). Global models tend to average out local variations and anomalies, resulting in a best-fit model for the whole area. 
The results of GWR are very similar to multiple regressions. Using the GWR tool available in ArcGIS we ran the analysis using the same explanatory variables that were found to be the best combination in the multi-linear regression from the above section. The GWR model was calibrated using known values for the dependent variable i.e. positive and negative arousal and known values for all the explanatory variables i.e. area with a 360 degree field of view, occlusivity with a 60 degree field of view, compactness with a 360 degree of field of view, occlusivity with a 360 degree field of view.

The statistical results show that AIC decreased and adjusted R-Squared values increased which indicates an improvement to the model. GWR produce a set of attributes that represent the coefficients for each explanatory variable. Figure 12 provides a visual representation of where the relationships between the explanatory variables and negative and positive arousal were strongest and weakest.

Figure 12 shows that for positive emotional arousal, the local correlation coefficients $r$ are always very small and max. 0.05 at the measurement points marked red in Figure 12a. This means that we cannot use our Isovist properties to explain positive arousal. We achieve a better result for the analysis of the relationship between negative arousal and Isovist properties. In Figure $12 \mathrm{~b}$ there are areas (with the measurement points marked in red) where we have local correlation coefficients r-squared of up to 0.32 . This indicates that in some places we can explain negative arousal by two Isovist properties by up to $32 \%$.

\section{INTERPRETATION}

In this section, we try and understand in more detail what the local correlation coefficients produced by GWR analysis can tell us, and in turn how we can interpret our results. For this, we must take a detailed look at the measured data and the result that we can predict with our model for negative emotional arousal(c.f. Formula (1)). In Figure 13 we have selected three areas (1-3) with a relatively large number of data points with high negative arousal values (Figure 13a). For these areas we look

Figure 12. Distribution of the local r-squared from the GWR analysis: a) Shows the distribution for positive arousal and b) The distribution for negative arousal. From the corresponding legend we can see, that the $r^{2}$ values are relevant for negative arousal in b only.
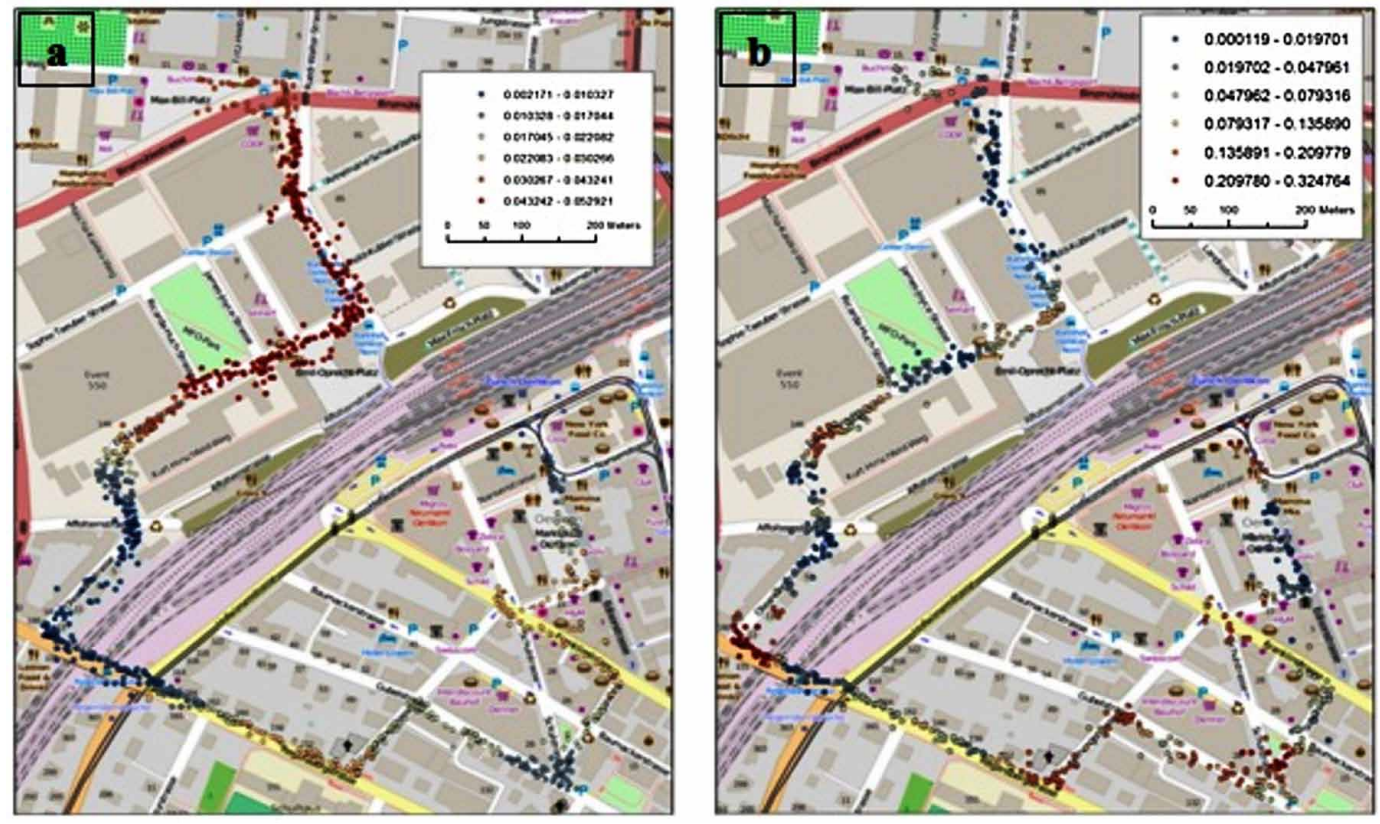
at the values predicted by the GWR model for each data point (Figure 13b). The predicted values are not as diverse as the measured ones but more equal to the values of the neighboring points. The predicted value changes along the path have more gradients and show clear clusters of values. This means our model results in a prediction of places with special characteristics - in our case places that tend to cause negative emotional arousal. Figure $13 \mathrm{c}$ provides an idea of how the coefficients from Figure $12 \mathrm{~b}$ are computed by the GWR model. The correlation coefficients are computed by looking at all pairs of observed and predicted values in a defined radius around a specific point.

A further interesting aspect of the our GWR result is that it has a high correlation coefficient at places where we have no negative emotional arousal. There is, for example, a high correlation shown in Figure 12b, which is marked as area 4 in Figure 13. Figure 13shows that we have very low negative emotional responses in this area, which is predicted well (around 30\%) by the GWR model. This means that the Isovist parameters are best at explaining why there are no negative emotional responses at a certain location.

The GWR model performs better at predicting negative arousal. Two Isovist parameters are used in our GWR model and the result has fluctuating local correlation coefficients ranging from $2 \%$ to $30 \%$ (Figure 13). There might be other physical parameters that influence people's arousal beyond the 2D planar parameters such as Isovist perimeter and occlusivity. Based on this observation, we propose the hypothesis that the GWR model works well if 2D planar parameters dominate in certain circumstance. For example, Figure 13b shows that the correlation coefficient is $30 \%$ around area 4 where we find very low negative emotional responses. When people walk through an area with many turnings and then reach 4,the degree of enclosure caused by surrounding buildings gradually decreases and the space becomes more open. The model we used represents the built environment in 2D only, We can, therefore, probably improve on it by including 3D Isovist parameters in a next step.

Figure 13. Comparison between observed (a) and predicted (b) negative arousal values and selected local $r^{2}$ coefficients (c). At c) the left bars show the observed and the middle bars the predicted negative emotional arousal values. The right bar shows the local correlation coefficient which is computed by looking at all pairs of observed and predicted values in a defined radius.




Another aspect that is interesting for understanding our results is how the Isovist parameters "perimeter" and "occlusivity" are related to the coefficients in Figure 12b, and how they are combined by our model (Equation (1)). We began by looking into the distribution of the measured values for each Isovist parameter in Figure 14a and $\mathrm{b}$. The values for perimeter and occlusivity seems to be highly correlated. But if we look at their influence on the prediction of negative emotional arousal expressed by the coefficients for both values in Figure 14c and d, we see that their effect for the GWR is very different. For example at area 2 in Figure 13, the coefficients shown in Figure 14c and $\mathrm{d}$ even have opposite signs. This clearly shows that we need both Isovist properties in our model for the prediction of negative emotional arousal.

Our main reason for introducing our statistical model was to investigate if there are certain spatial configurations which have a tendency to produce specific emotional responses. These areas are located at places where the spatial situation changes somehow, e.g. as a result of turning a corner. The GWR model was able to reflect at least some aspects of the dynamic changes of the spatial situations along the path.

With the GWR model we were able to show that we can explain negative arousal at the places marked in Figure 13 between $15 \%$ and $30 \%$ by using two Isovist properties. For predicting emotional responses for new spatial configurations we would need a global linear regression model. Unfortunately, since the required model is global in nature, it is not possible to accurately characterize the relationship between the independent variable that we want to predict (negative emotional arousal) and the dependent variables (Isovist parameters), because the relationship is positive in some portions of the study region and negative in others (Figure 12b).

The fact that we can show only a relationship between negative emotional response and location corresponds to the findings of the study from Hogertz's (2010). This relationship seems to be present

Figure 14. Computed Isovist properties and the corresponding coefficients for the GWR model: a) Shows the computed Isovist values for perimeter for a $360^{\circ}$ field of view and b) Show the computed Isovist values for occlusivity for a $60^{\circ}$ field of view. c) Shows the coefficient for perimeter $360^{\circ}$ and d) For occlusivity $60^{\circ}$ for the GWR model.

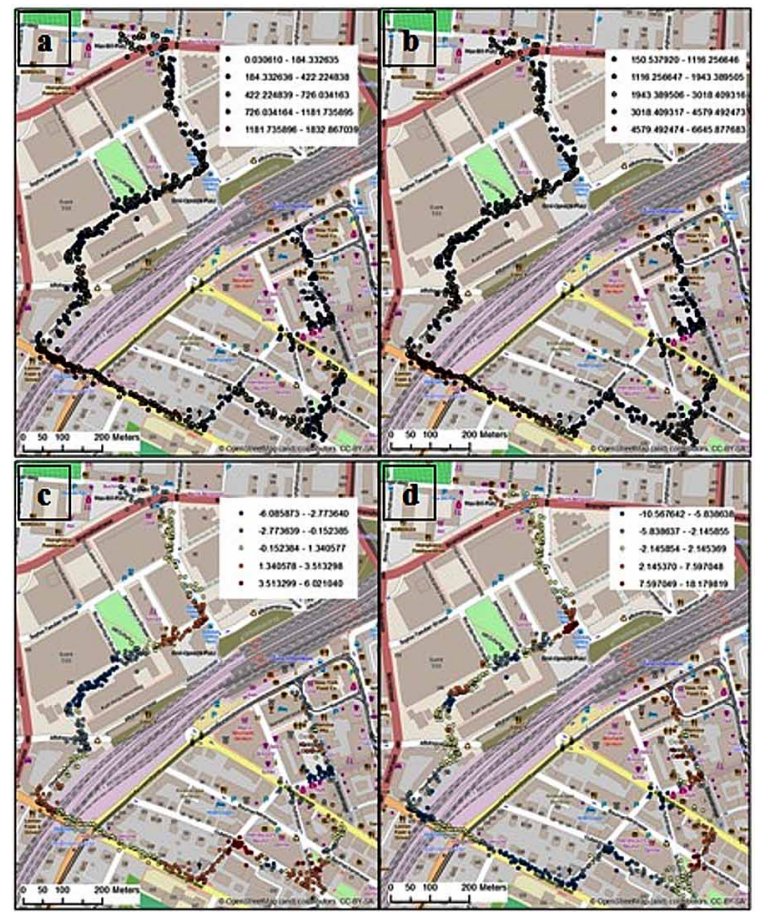


for the very different characteristics of the respective routes used in the different cities as well as the different time of day at which the study was carried out: Hogertz's (2010) study was done at night, ours during the day.

\section{CONCLUSION AND OUTLOOK}

We started by introducing the idea of a computationally aided evidence-informed design process for the design of urban environments, noting that it would be useful to have methods that help predict the most-likely behavioral responses of a specific design proposal. In this study we investigated the emotional responses of pedestrians along a predefined path. By capturing emotional responses in a real environment using a Smartband, locating this data via GPS and clustering the located data by means of spatial statistics, we have found that there are locations on the predefined path, where different test persons exhibit the same degree of positive or negative arousal. This is crucial for the further development of CEID, because urban design decisions generally affect a large number of different people.

On closer examination of these locations we continued with the hypothesis that changes between certain spatial situations probably cause the highest emotional responses. This was supported for negative emotional arousal by a GWR model, which explained local values up to $30 \%$. The model we developed can be used to explain negative emotional arousal using the two Isovist parameters "perimeter" and "occlusivity". Unfortunately, the GWR model cannot reliably make predictions about whether a certain new spatial configuration will produce negative emotional arousal. We, therefore, need to investigate more sophisticated time series analyses and to collect a much larger data set for analysis.

The outcome of the presented study provides the motivation for undertaking a larger study in order to obtain statistically significant results as the sample sizes of our study and all related studies mentioned earlier are too small. In addition, in a forthcoming study we want to control environmental effects by incorporating sensors for noise, temperature, and air quality and to record what the test persons see.

\section{ACKNOWLEDGMENT}

We are indebted to Dr. Georgios Papastefanou from Bodymonitor for his advice during our discussions, his technical assistance in the use of the Smartband, and for interpreting the data. This research project was funded by the Swiss National Science Foundation (project number 100013L_149552) and National Natural Science Foundation of China (No. 51408442) partially by the Swiss Government Excellence Scholarships for Foreign Scholars and Artists. 


\section{REFERENCES}

Academy of Neuroscience for Architecture. (2013). Retrieved from http://www.anfarch.org/

Batty, M. (2001). Exploring Isovist Fields: Space and Shape in Architectural and Urban Morphology. Environment and Planning. B, Planning \& Design, 28(1), 123-150. doi:10.1068/b2725

Benedikt, M. L. (1979). To take hold of space: Isovists and Isovist fields. Environment and Planning. B, Planning \& Design, 6(1), 47-65. doi:10.1068/b060047

Bergner, B. S., Exner, J., \& Memmel, M. (2013). Human Sensory Assessment Methods in Urban Planning: A Case Study in Alexandria. In M. Schrenk, V Popovich, P Zeile, P., Elisei (Ed.), Proceedings of REAL CORP 2013, (pp. 20-33). Rome, Italy.

Bergner, B. S., Zeile, P., \& Papastefanou, G. (2011). Emotional Barrier-GIS: A new Approach to Integrate Barrier-Free Planning in Urban Planning Processes. In M. Schrenk, V. Popvich, \& P. Zeile (Eds.), REAL CORP 20 (pp. 247-257). Essen.

Bittermann MS, Ciftcioglu O. (2008). Visual Perception Model for Architectural Design. Journal of Design Research, 7(1), 35-60.

Burnham, K. P., \& Anderson, D. R. (2002). Model Selection and Multimodel Inference: a practical informationtheoretic approach (2nd ed.). New York: Springer.

Chong, G. H., Brandt, R., \& Martin, W. M. (2010). Design Informed: Driving Innovation with Evidence-Based Design. Wiley.

Compagnon, R. (2004). Solar and daylight availability in the urban fabric. Energy and Building, 36(4), 321-328. doi:10.1016/j.enbuild.2004.01.009

Conroy-Dalton, R., Hölscher, C., Peck, T., \& Pawar, V. (2010). Judgements of building complexity \&navigability in virtual reality. In the Proceedings of (pp. 49-64). Work. Spat. Cogn.

Edelstein, E. A., Gramann, K., \& Schulze, J. (2008). Neural Responses during Navigation in the Virtual Aided Design Laboratory Brain Dynamics of Orientation in Architecturally Ambiguous Space. In C. Hölscher \& S. Torgrude (Eds.), Rep. Ser. Transreg. Collab. Res. Cent. SFB/TR 8 Spat (pp. 35-41). Cogn.

Edelstein, E. A., \& Macagno, E. (2012). Sustainable Environmental Design in Architecture: Impacts on Health. In S.T. Rassia, \& P.M. Pardalos (Ed), Sustainable. Environment Design Architecture (pp. 27-41). New York, NY, USA: Springer.

Fotheringham, A. S., Brunsdon, C., \& Charlton, M. (2003). Geographically weighted regression: the analysis of spatially varying relationships. John Wiley \& Sons.

Franz, G., \& Wiener, J. M. (2008). From space syntax to space semantics: A behaviorally and perceptually oriented methodology for the efficient description of the geometry and topology of environments. Environment and Planning. B, Planning \& Design, 35(4), 574-592. doi:10.1068/b33050

Gehl, J. (2010). Cities for People Washington - Covelo - London, Island Press.

Getis, A. (1991). Spatial interaction and spatial autocorrelation: A cross product approach. Environment \& Planning A, 23(9), 1269-1277. doi:10.1068/a231269

Hillier, B. (1996). Space is the machine: a configurational theory of architecture. Cambridge: Cambridge University Press.

Hillier, B., \& Iida, S. (2005). Network and psychological effects in urban movement. Proceedings of the 5th Int. Symposium Space Syntax (pp. 475-490).

Hogertz, C. (2010). Emotions of the urban pedestrian: sensory mapping. In W.-R. Nickel, \& W. Vermeulen (Eds.), PQN Final Report - Part B4 (WALK21) (pp. 31-52).

Mavros, P., Coyne, R., Roe, J., \& Aspinall, P. (2012). Engaging the Brain: Implications of mobile EEG for spatial representation. Proceedings of 30th eCAADe Conf. Molab, Prague, Czech Republic (pp. 647-656). 
Meilinger, T., Franz, G., \& Bülthoff, H. H. (2012). From Isovists via mental representations to behaviour: First steps toward closing the causal chain. Environment and Planning. B, Planning \& Design, 39(1), 48-62. doi:10.1068/b34048t

Mitchell, A. (2005). The ESRI Guide to GIS Analysis (Vol. 2). Redlands, Ca, USA: ESRI Press.

Papastefanou, J. (2013a). ExperimentelleValidierungeines Sensor-ArmbandeszurmobilenMessungphysiologis cher Stress- Reaktionen. GESIS-Technical Reports, 07, 14.

Papastefanou, J. (2015). Bodymonitor: Sensing emotions. Retrieved from http://www.bodymonitor.de/ Projekte $\% 28$ neu $\% 29$

Peters, D., \& Richter, K. (2008). Taking off to the Third Dimension: Schematization of Virtual Environments. International Journal of Spatial Data Infrastructures Research, 3, 20-37.

Rittel, H. W. J., \& Webber, M. M. (1973). Dilemmas in a General Theory of Planning. Policy Sciences, 4(2), 155-169. doi:10.1007/BF01405730

Roe, J. J., \& Aspinall, P. a, Mavros, P., \& Coyne, R. (2013). Engaging the Brain: The Impact of Natural versus Urban Scenes Using Novel EEG Methods in an Experimental Setting. Environmental Sciences, 1(2), $93-104$.

Russell, J. A. (1980). A circumplex model of affect. Journal of Personality and Social Psychology, 39(6), 1161-1178. doi:10.1037/h0077714

Sevtsuk, A. (2010). Path and place : a study of urban geometry and retail activity in Cambridge and Somerville. MA: Massachusetts Institute of Technology.

Stähli, J., \& Keller, S. (2013). PräzisePositionsbestimmungmit Low-Cost-GPS und Postprocessing. http://eprints. hsr.ch/270/1/TR-1301-IFS-HSR_v5.pdf

Stamps, A. E. (2009). On Shape and Spaciousness. Environment and Behavior, 41(4), 526-548. doi:10.1177/0013916508317931

Turner, A., \& Penn, A. (1999). Making Isovists syntactic: Isovist integration analysis. Retrieved from http:// discovery.ucl.ac.uk/87836/

Wiener, J. M., Hölscher, C., Büchner, S. J., \& Konieczny, L. (2012). Gaze Behaviour during Space Perception and Spatial Decision Making. Psychological Research, 76(6), 713-729. doi:10.1007/s00426-011-0397-5 PMID:22139023

Winkielman, P., Berridge, K. C., \& Sher, S. (2011). Emotion, Consciousness, and Social Behavior. In J. Decety \& J. T. Cacioppo (Eds.), The Oxford Handbook of Social Neuroscience (pp. 195-211). Oxford University Press.

Zajonc, R. B. (1984). On the primacy of affect. The American Psychologist, 39(2), 117-123. doi:10.1037/0003066X.39.2.117 